\title{
Limitations of screening for hepatocellular carcinoma
}

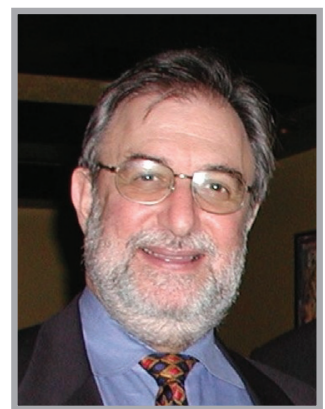

Morris Sherman* “...not all patients who develop hepatocellular carcinoma while undergoing screening have a good outcome ... and a significant proportion of these patients will still die either from their

hepatocellular carcinoma or from the underlying liver disease."
Hepatocellular carcinoma (HCC) screening has become common practice and has been shown to be highly successful in good hands. In Japan, more than $60 \%$ of all HCCs are discovered when they are smaller than $2 \mathrm{~cm} \mathrm{[1].} \mathrm{In} \mathrm{Europe,} \mathrm{one} \mathrm{report} \mathrm{indi-}$ cates that $74 \%$ of screen-detected HCCs are smaller than $3 \mathrm{~cm}[2]$. These are easily treated and can be cured. However, not all patients who develop HCC while undergoing screening have a good outcome. A significant proportion of these patients will still die either from their HCC or from the underlying liver disease.

Many authors have emphasized that screening consists of more than just tests. There should be a process that includes identification of the at-risk group(s), application of the test, appropriate recall procedures and a process in place to manage diseases discovered by screening. For best results screening should be carried out as a controlled program, rather than on an ad hoc basis.

Thus, failure of HCC screening can be looked at from the point of view of the program, or from the point of view of the individual who suffered a failure of screening.
Failure of screening in an individual patient essentially means failure of the screening tests to detect the HCC at a curable stage or failure to properly interpret the screening test results (recall procedures), or failure to apply the appropriate treatment for that stage of disease at a timely interval after detection.

Studies of resection and local ablation suggest that the likelihood of cure starts to diminish once the HCC is between 2 and $2.5 \mathrm{~cm}$ in diameter [3-5]. Therefore, the target size lesion that screening should detect is below approximately $2.5 \mathrm{~cm}$. To be useful, a screening test should be able to find most lesions smaller than this size. Failure of the screening test to identify such a small cancer that is present and may be curable may be due to inadequate sensitivity of the screening test, too long an interval between screening tests, development of an aggressive cancer that exhibits microvascular invasion and/or metastasis even while still small, or to an infiltrative tumor that does not form a clearly identifiable lesion.

The serological screening tests are AFP concentration, des- $\gamma$-carboxy prothrombin and the L3 fraction of AFP. However,

*Department of Medicine, University of Toronto, Toronto General Hospital, 9N985, 200 Elizabeth Street, Toronto, ON, M5G 2C4, Canada; Tel.: +1 416340 4756; Fax: +1 46591 2107; morris.sherman@uhn.on.ca

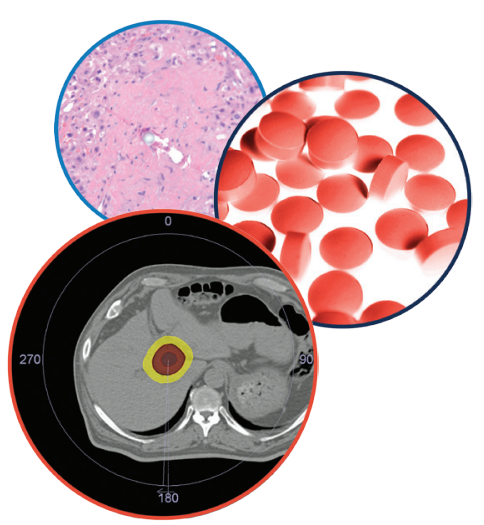

"Studies of resection and local ablation suggest that the likelihood of cure starts to diminish once the hepatocellular carcinoma is between 2 and $2.5 \mathrm{~cm}$ in diameter."

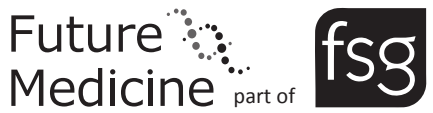


"Screening programs fail when the population being screened does not have a risk high enough to warrant screening." many studies have shown that these are insufficiently sensitive tests, picking up no more than approximately $60 \%$ of small HCCs [6-15]. As the HCC gets larger the likelihood of any of the tests being elevated increases, but even at a tumor size of $>5 \mathrm{~cm}$ the sensitivity is less than optimal. AFP is the most commonly used, but is the least sensitive. Furthermore, all three of these tests also identify advanced HCC with a poor prognosis. A test that indicates advanced disease cannot also be a marker of early-stage disease.

The recommended screening test is ultrasound. The sensitivity of ultrasound in a noncirrhotic liver is better than $80 \%$ [16], but for small HCC the sensitivity falls to approximately $60 \%$. There is some controversy as to whether the addition of biomarkers to ultrasound enhances detection. Studies in Japan using ultrasound and biomarkers have approximately the same detection rate as in Europe without biomarkers [1,2].

There are well-known factors that limit the sensitivity of ultrasound, most importantly, fatty liver, with or without obesity. The presence of fat attenuates the ultrasound beam, so that the beam is less able to penetrate the full substance of the liver. Typically, the parts of the liver most distant from the probe - that is, posteriorly and superiorly - are not well seen. Cirrhosis, with the presence of multiple nodules, also makes it difficult to determine whether any of the nodules are suspicious for HCC.

Finally, good ultrasound is technically demanding. Cursory examination will miss important lesions. Poor technique is probably the most important cause of failure of ultrasound to detect the presence of a significant nodule. However, CT scan or MRI is not the answer. Although these are more sensitive than ultrasound and do not suffer from the problems of fat or cirrhosis, they are not suitable for HCC screening. CT scan without contrast is probably not more sensitive than ultrasound, and may even be less sensitive. If only arterial or venous phase imaging is performed important diagnostic lesion characteristics will be missed. For best sensitivity and specificity a four-phase CT scan is required [17]. Thus, screening would entail essentially four CT scan runs twice a year, possibly for many years. The radiation dose would be significant. We worry less about the radiation dose in patients who have HCC, but in patients not known to have HCC and who may never get $\mathrm{HCC}$ it is questionable whether the radiation dose is justified. MRI does not involve radiation.
More recently, MRI hepatobiliary phase imaging contrast agents have been used for HCC diagnosis. The value of these agents as screening tests has not been evaluated. However, both CT scan and MRI are very costly, with a very high incremental cost-efficacy ratio, making them economically unfeasible [18].

Irregular screening is another cause of failure to detect small HCC. The ideal interval between screening tests is not known. The interval depends on the time it takes for a tumor to grow from being undetectable to being incurable. Since growth rates vary, and neither curability of small lesions, nor incurability of larger lesions are $100 \%$ predictable, identification of the appropriate screening interval for an individual patient is not possible. However, studies have suggested that a 6-month interval is the most appropriate balance between too many false positives and too many missed opportunities for cure.

Another reason for failure of screening at a personal level is failure to adequately investigate screen-detected lesions. This surprisingly common. Physicians may not immediately investigate screen-detected lesions, preferring to monitor progress. Decision analysis, however, has indicated that this is the least effective option [19]. The analysis found that a delay in the investigation of screen-detected lesions increased the risk of diagnosis at a stage associated with less than optimal cure rates.

The final reason for failure of HCC screening at an individual level is failure to offer appropriate treatment. This is uncommon with small HCC, which approximately responds equally well to local ablation, resection or transplantation. However, detection of HCC at a later stage (e.g., $>2 \mathrm{~cm}$ ) is less forgiving if the wrong treatment is offered. Local ablation is less effective at these tumor sizes, and resection or transplantation might offer better survival. However, local ablation, usually radiofrequency ablation, continues to be offered to such patients.

Screening programs fail when the population being screened does not have a risk high enough to warrant screening. Although groups at risk for HCC have been defined, the majority of people in those groups will not develop HCC. More recently, risk scores have been developed that allow identification of those at lower risk within the larger at-risk groups, who can be withdrawn from screening programs.

Screening programs will also fail if overdiagnosis is prevalent - that is, the diagnosis of a 
lesion as cancer when it is unlikely to cause the patient's death. Overdiagnosis is an inevitable consequence of screening, but it is likely that overdiagnosis plays only a small role in HCC screening. Furthermore, the algorithm described in the American Association for the Study of Liver Diseases guidelines [20], and which has been validated [21-23], minimizes the likelihood of overdiagnosis by requiring either histology or classical radiological appearances of HCC for confirmation of the diagnosis.

Therefore, it can be seen that screening for HCC may fail on several levels. It is easy to understand failure of screening of an individual patient, but factors leading to failure of a screening program are less well recognized, but equally as important.

\section{Financial \& competing interests disclosure}

The author has no relevant affliations or financial involvement with any organization or entity with a financial interest in or financial conflict with the subject matter or materials discussed in the manuscript. This includes employment, consultancies, honoraria, stock ownership or options, expert testimony, grants or patents received or pending, or royalties.

No writing assistance was utilized in the production of this manuscript.

\section{References}

1 Izumi N. Diagnostic and treatment algorithm of the Japanese society of hepatology: a consensus-based practice guideline. Oncology 78(Suppl. 1), 78-86 (2010).

2 Trinchet JC, Chaffaut C, Bourcier V et al. Ultrasonographic surveillance of hepatocellular carcinoma in cirrhosis: a randomized trial comparing 3-and 6-month periodicities. Hepatology 54, 1987-1997 (2011).

3 Ikai I, Arii S, Kojiro M et al. Reevaluation of prognostic factors for survival after liver resection in patients with hepatocellular carcinoma in a Japanese nationwide survey. Cancer 101(4), 796-802 (2004).

4 Shah SA, Cleary SP, Wei AC et al. Recurrence after liver resection for hepatocellular carcinoma: risk factors, treatment, and outcomes. Surgery 141(3), 330-339 (2007).

5 Sala M, Llovet JM, Vilana R et al.; Barcelona Clínic Liver Cancer Group. Initial response to percutaneous ablation predicts survival in patients with hepatocellular carcinoma. Hepatology 40(6), 1352-1360 (2004).

6 Farinati F, Marino D, De Giorgio M et al. Diagnostic and prognostic role of alphafetoprotein in hepatocellular carcinoma: both or neither? Am. J. Gastroenterol. 101(3), 524-532 (2006).

7 Trevisani F, D'Intino PE, Morselli-Labate AM et al. Serum alpha-fetoprotein for diagnosis of hepatocellular carcinoma in patients with chronic liver disease: influence of $\mathrm{HBsAg}$ and anti-HCV status. J. Hepatol. 34(4), 570-575 (2001).

8 Tong MJ, Blatt LM, Kao VW. Surveillance for hepatocellular carcinoma in patients with chronic viral hepatitis in the United States of America. J. Gastroenterol. Hepatol. 16(5), 553-559 (2001).
9 Gambarin-Gelwan M, Wolf DC, Shapiro R, Schwartz ME, Min AD. Sensitivity of commonly available screening tests in detecting hepatocellular carcinoma in cirrhotic patients undergoing liver transplantation. $\mathrm{Am}$. J. Gastroenterol. 95(6), 1535-1538 (2000).

10 Sterling RK, Jeffers L, Gordon F et al. Utility of Lens culinaris agglutinin-reactive fraction of alpha-fetoprotein and des-gamma-carboxy prothrombin, alone or in combination, as biomarkers for hepatocellular carcinoma. Clin. Gastroenterol. Hepatol. 7(1), 104-113 (2009).

11 Sterling RK, Jeffers L, Gordon F et al. Clinical utility of AFP-L3\% measurement in North American patients with HCV-related cirrhosis. Am. J. Gastroenterol. 102(10), 2196-2205 (2007).

12 Marrero JA, Feng Z, Wang Y et al. Alphafetoprotein, des-gamma carboxyprothrombin, and lectin-bound alpha-fetoprotein in early hepatocellular carcinoma. Gastroenterology 137(1), 110-118 (2009).

13 Sterling RK, Wright EC, Morgan TR et al. Frequency of elevated hepatocellular carcinoma (HCC) biomarkers in patients with advanced hepatitis C. Am. J. Gastroenterol. 107(1), 64-74 (2012).

14 Lok AS, Sterling RK, Everhart JE et al.; HALT-C Trial Group. Des-gamma-carboxy prothrombin and alpha-fetoprotein as biomarkers for the early detection of hepatocellular carcinoma. Gastroenterology 138(2), 493-502 (2010).

15 Singal A, Volk ML, Waljee A et al. Meta-analysis: surveillance with ultrasound for earlystage hepatocellular carcinoma in patients with cirrhosis. Aliment Pharmacol. Ther. 30(1), 37-47 (2009).

16 Singal AG, Nehra M, Adams-Huet B et al. Detection of hepatocellular carcinoma at advanced stages among patients in the
HALT-C trial: where did surveillance fail? Am. J. Gastroenterol. 108(3), 425-432 (2013).

17 Furlan A, Marin D, Vanzulli A et al. Hepatocellular carcinoma in cirrhotic patients at multidetector CT: hepatic venous phase versus delayed phase for the detection of tumour washout. Br. J. Radiol. 84(1001), 403-412 (2011)

18 Andersson KL, Salomon JA, Goldie SJ, Chung RT. Cost effectiveness of alternative surveillance strategies for hepatocellular carcinoma in patients with cirrhosis. Clin. Gastroenterol. Hepatol. 6(12), 1418-1424 (2008).

19 Bremner KE, Bayoumi AM, Sherman M, Krahn MD. Management of solitary $1 \mathrm{~cm}$ to $2 \mathrm{~cm}$ liver nodules in patients with compensated cirrhosis: a decision analysis. Can. J. Gastroenterol. 21, 491-500 (2007).

20 Bruix J, Sherman M. Management of hepatocellular carcinoma: an update. Hepatology 53, 1020-1022 (2011).

21 Furlan A, Marin D, Cabassa P et al. Enhancement pattern of small hepatocellular carcinoma (HCC) at contrast-enhanced US (CEUS), MDCT, and MRI: intermodality agreement and comparison of diagnostic sensitivity between 2005 and 2010 American Association for the Study of Liver Diseases (AASLD) guidelines. Eur. J. Radiol. 81(9), 2099-2105 (2012).

22 Khalili K, Kim TK, Jang HJ, Yazdi LK, Guindi M, Sherman M. Indeterminate 1-2-cm nodules found on hepatocellular carcinoma surveillance: biopsy for all, some, or none? Hepatology 54(6), 2048-2054 (2011).

23 Sangiovanni A, Manini MA, Iavarone $M$ et al. The diagnostic and economic impact of contrast imaging techniques in the diagnosis of small hepatocellular carcinoma in cirrhosis. Gut 59(5), 638-644 (2010). 